\title{
Observations of the $\mathrm{CH}_{3} \mathrm{OH} 4_{2}-5_{1}$ E Line Toward the Sgr B2 Region
}

\author{
Young Chol Minh ${ }^{1 \dagger}$ and Sang Joon Kim ${ }^{2}$
}

${ }^{1}$ Korea Astronomy and Space Science Institute, Daejeon 305-348, Korea

${ }^{2}$ School of Space Research, Kyunghee University, Yongin 446-701, Korea

The $\mathrm{CH}_{3} \mathrm{OH} 4_{2}-5_{1}$ E transition was observed toward the Sgr B2 region, including the Principal Cloud and its surroundings. This methanol transition shows an extended emission along the 2'N cloud, which is believed to be colliding with the Principal Cloud and may trigger the massive star formation in this cloud. This extended methanol emission may also suggest that the ${ }^{\prime} \mathrm{N}$ cloud is under shocks. We derive total methanol column density $\mathrm{N}\left(\mathrm{CH}_{3} \mathrm{OH}\right)=2.9 \pm 0.3 \times 10^{14} \mathrm{~cm}^{-2}$ toward the peak position of the extended emission. The fractional abundance of methanol is about $10^{-9}$, relative to the estimated total $\mathrm{H}_{2}$ abundance, which is similar to the methanol abundances in quiet gas phase.

Keywords: interstellar matter (ISM), ISM abundances, ISM individual (Sgr B2), ISM molecules

\section{INTRODUCTION}

The Sgr B2 region in our Galactic center contains giant molecular clouds, which represent extreme cases of high luminosity star formation. The gas in the Sgr B2 region is mainly concentrated in a dense central component, "the Principal Cloud," 5-10 pc in diameter with a relatively high mean density, $\mathrm{n}\left(\mathrm{H}_{2}\right) \approx 1 \times 10^{5} \mathrm{~cm}^{-3}$ (Irvine et al. 1987, Minh et al. 1998). Along the north-south direction there exist three prominent star-forming cores 'inside' the Principal Cloud, Sgr B2 (N), (M), and (S) which include clusters of compact HII regions having newly born massive stars. The density and temperature structures of this region are extremely complicated (Martìn-Pintado et al. 1999, Rodrìguez-Fernàndez et al. 2002, and references therein). The massive star formations in the core of the Principal Cloud has been suggested to be triggered by cloud collisions (Hasegawa et al. 1994, Mehringer et al. 1995, Oka et al. 1998, Sato et al. 2000) although there exist many difficulties in identifying different gas components by using the optically thick lines. The latest star formation may be occurring at the 2 arcmin north $\left(2^{\prime} \mathrm{N}\right)$ position from Sgr B2 (M), where the isocyanic acid (HNCO) emission peaks probably resulting from the collision between the Principal Cloud and the 2'N cloud (Minh et al. 1998, Minh \& Irvine 2006). We observed one of the methanol transitions in the $200 \mathrm{GHz}$ band toward the Sgr B2 molecular cloud using the Swedish-European Southern Observatory (ESO) submillimeter telescope (SEST) in Chile, and discuss the morphology of its emission in this region.

Methanol is a slightly asymmetric rotor with hindered internal rotation and significant $\mathrm{a}$ - and $\mathrm{b}$-axis dipole moments $\left(\mu_{\mathrm{a}}=0.885 \mathrm{D}, \mu_{\mathrm{b}}=1.44 \mathrm{D}\right)$, and has a complex spectrum that is sensitive to interstellar conditions. The modeling of methanol excitation has been known to be extremely difficult. Even when the lines are not masing, they may be expected to be distinctly non-LTE excitation, except under the highest density conditions, where collisions could thermalize the level populations (Leurini et al. 2004, 2007). Methanol $\left(\mathrm{CH}_{3} \mathrm{OH}\right)$ is thought to form mainly on the grain surface in dense cores by the hydrogenation of $\mathrm{CO}$, and observed to be one of the major solid components in dust ice mantles (Dartois et al. 1999, Gibb 2000). Methanol is the predominant $\mathrm{O}$-bearing species in the gas phase after evaporation from dust grains, and the

(c) This is an Open Access article distributed under the terms of the Creative Commons Attribution Non-Commercial License (http://creativecommons.org/licenses/by-nc/3.0/) which permits unrestricted non-commercial use, distribution, and reproduction in any medium, provided the original work is properly cited.

Received Jan 20, 2011 Revised Feb 03, 2011 Accepted Feb 11, 2011 ${ }^{\dagger}$ Corresponding Author

E-mail:minh@kasi.re.kr

Tel: +82-42-865-3263 Fax: +82-42-865-2020 
key species which initiates the early phase oxygen chemistry in hot cores (Rodgers \& Charnley 2003). In addition to the fact that methanol masers are often found abundant very close to young stellar objects, its thermal emission is thought to unambiguously trace hot cores and/or outflows associated with massive star formation (Minh et al. 1993, Minier et al. 2000).

Various methanol lines have been detected toward the Sgr B2 molecular cloud, especially, toward the (M) and $(\mathrm{N})$ positions of the Principal Cloud (Cummins et al. 1986, Mehringer \& Menten 1997, Nummeline et al. 2000). In this paper, we report the observational results of the $\mathrm{CH}_{3} \mathrm{OH} 4_{2}-5_{1} \mathrm{E}$ transition toward the Sgr B2 region, and discuss the emission properties in this cloud. Our study is mainly focused on the morphology of this emission associated with the 2'N Cloud, which is claimed to be colliding with the main gas concentration in Sgr B2, the Principal Cloud (Minh et al. 1998, Minh \& Irvine 2006).

\section{OBSERVATIONS}

Observations were carried out using the $15 \mathrm{~m} \mathrm{SEST}^{1}$ (Booth et al. 1989) on La Silla, Chile, in April 2000. The methanol $\left(\mathrm{CH}_{3} \mathrm{OH}\right) 4_{2}-5_{1} \mathrm{E}$ transition at $216.95 \mathrm{GHz}$ was observed using a dual channel SIS receiver during the survey of various molecular lines toward our Galactic center region (Minh et al. 2005, Minh \& Irvine 2006). Two 1,500 channel acousto-optic spectrometers with channel spacing of $0.69 \mathrm{MHz}$ were used but the frequency resolution of the system was approximately 1.2 MHz. The halfpower beamwidth (HPBW) and main beam efficiency are 22 " and 0.60 , respectively, at $220 \mathrm{GHz}$. Spectra were taken with the position switching mode using the reference position $(\mathrm{l}, \mathrm{b})=\left(0.0^{\circ}, 2.5^{\circ}\right)$, and the map was made with a grid of 2 arcmin. The antenna temperatures $\mathrm{T}_{\mathrm{A}}^{*}$ quoted in this paper have been corrected for antenna and atmospheric losses by means of the standard chopper wheel method, but not for possible beam dilution. The typical system temperatures were about 220-240 K (SSB) and typical rms $(1 \sigma)$ of the spectra are $\sim 120 \mathrm{mK}$.

\section{RESULTS AND DISCUSSION}

Fig. 1 shows a spectrum observed toward the Sgr B2 (M) position. The line intensity appears to be strongly

${ }^{1}$ SEST is operated by Onsala Space Observatory, the Swedish National Facility for Radio Astronomy, with financial support from the Swedish Natural Science Research Council (NFR) and ESO. enhanced at this (M) position, relative to other observed points in this region, which is probably associated with massive star formation. This methanol line seems to have a broad wing component $\left(\Delta \mathrm{v} \geq 30 \mathrm{~km} \mathrm{~s}^{-1}\right)$, but higher spatial resolution observations are necessary for further studies. The velocity integrated intensity map of the observed methanol line is shown in Fig. 2. The location of Sgr B1 is indicated with a filled square in the figure, which is an HII region complex (LaRosa et al. 2000). Sgr B1 is a part of the Ridge connecting the Galactic center radio Arc and the Sgr B2 cloud (Lis et al. 2001), which shows very weak molecular line emissions in our observations. In this paper we focus on the gas cloud associated with Sgr B2 (M) and its surroundings.

As shown in Fig. 2 the velocity integrated intensity of the $4_{2}-5_{1}$ line of methanol shows highly enhanced toward the (M) position, compared to other observed points in this region. The high enhancement of this methanol emission line, which appears to be unresolved in this 22 " beam, is thought to result from interactions between the hot cores and outflows from massive stars forming as a cluster in this region. The morphology of this extended emission shows a very close correlation with the 2'N cloud shown in Fig. 2 (Minh \& Irvine 2006). The 2'N cloud, as a part of a large-scale "Ring" component (radius $\sim 5 \mathrm{pc}$ ), may be experiencing the very first phase of collapse by colliding with the Principal Cloud, which could have resulted in the high activities of star formation in the Principal Cloud (Minh et al. 1998, Minh \& Irvine 2006). Although the gas component in the Sgr B2 region is concentrated mainly in the Principal Cloud, we found no extra/extended emission toward the other part of the Principal Cloud at the observed sensitivity. It means that the dense

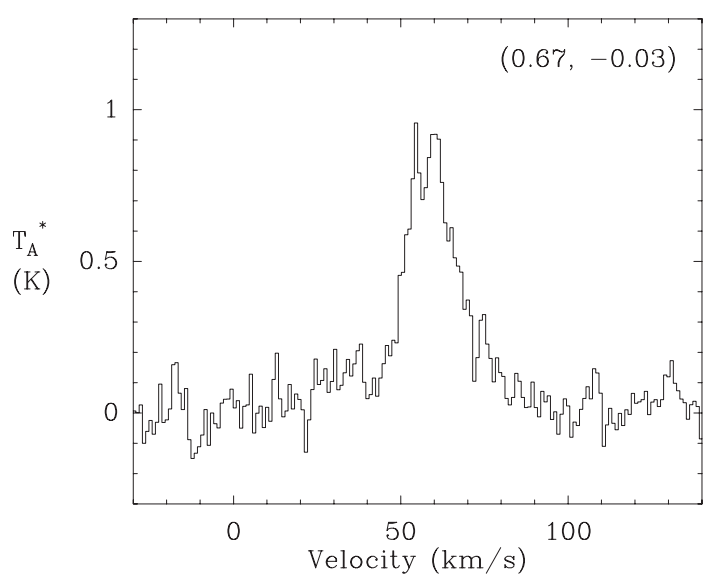

Fig. 1. Spectrum of the $\mathrm{CH}_{3} \mathrm{OH} 4_{2}-5_{1}$ line, obtained toward $\left(0.67^{\circ},-0.03^{\circ}\right)$ in galactic coordinate, which is roughly the Sgr B2 (M) position. 
gas in the Principal Cloud is relatively quiet except the star-forming cores in this cloud (Irvine et al. 1987). On the other hand the relatively weak but extended emission of methanol toward the 2 ' $\mathrm{N}$ cloud may suggest that this cloud is under shocks, which makes the solid methanol in the grain mantles evaporate into the gas phase.

We estimate the total column density of methanol, by assuming optically thin emission and an LTE condition (Irvine et al. 1987), $\mathrm{N}\left(\mathrm{CH}_{3} \mathrm{OH}\right)=1.2 \pm 0.1 \times 10^{15} \mathrm{~cm}^{-2}$ toward $(\mathrm{M})$, and $2.9 \pm 0.3 \times 10^{14} \mathrm{~cm}^{-2}$ toward the peak position of the extended emission. The errors come from the range of rotational temperature applied, $10-20 \mathrm{~K}$, and the observed spectral noise $(1 \sigma)$ gives about $\leq 5 \%$ and $~ 30 \%$ further uncertainty, for the values obtained toward (M) and the peak of the extended emission, respectively. Galactic methanol abundances are known to be about $10^{-9}$ relative to $\mathrm{H}_{2}$ for quiet dense clouds, but in smaller scales, methanol can be enhanced by factors of a few hundred over dark cloud values in shocks and outflows (Meier \& Turner 2005). The total $\mathrm{H}_{2}$ column density, $\mathrm{N}\left(\mathrm{H}_{2}\right)$, is highly uncertain, but we assume that $\left.\mathrm{N}_{(\mathrm{H}}\right) \approx 1 \times 10^{24} \mathrm{~cm}^{-2}$ toward $(\mathrm{M})$ and one-fourth of that toward the 2 ' $\mathrm{N}$ position (Irvine et al. 1987, Minh et al. 1998). Then we derive the "beam-averaged" fractional abundance of methanol, relative to the total $\mathrm{H}_{2}$ abundance, to be about $10^{-9}$ for both Sgr B2 (M) and the peak of the extended emission. However, since the emission toward Sgr B2 (M) is unresolved in our beam, the fractional abundance of methanol will be much higher at the actual methanol core. And the estimated total $\mathrm{H}_{2}$ column density toward the 2' $\mathrm{N}$ position includes the contribution from the Principal Cloud, which is probably significant. For better estimation of the methanol abundances, we need higher spatial resolution observations and multi-transition methanol observations, in addition to a better derivation of the total $\mathrm{H}_{2}$ column density for separate cloud components in the Sgr B2 region.

\section{SUMMARY}

We have observed the $\mathrm{CH}_{3} \mathrm{OH} 4_{2}-5_{1}$ transition toward the Sgr B2 region, including the Principal Cloud and its surroundings. This methanol emission shows a strong enhancement toward Sgr B2 (M), where massive stars are forming as a cluster. The low intensity emission shows to be extended mainly along the 2'N cloud, which was suggested to be colliding with the Principal Cloud (Minh et al. 1998, Minh \& Irvine 2006). The methanol emission distribution confirms that the 2' $\mathrm{N}$ cloud is under shocks, assuming that shock makes the solid methanol in the grain mantles evaporate into gas phase. We derive total methanol column density $\mathrm{N}\left(\mathrm{CH}_{3} \mathrm{OH}\right)=2.9 \pm 0.3 \times 10^{14}$ $\mathrm{cm}^{-2}$ toward the peak position of the extended emission.

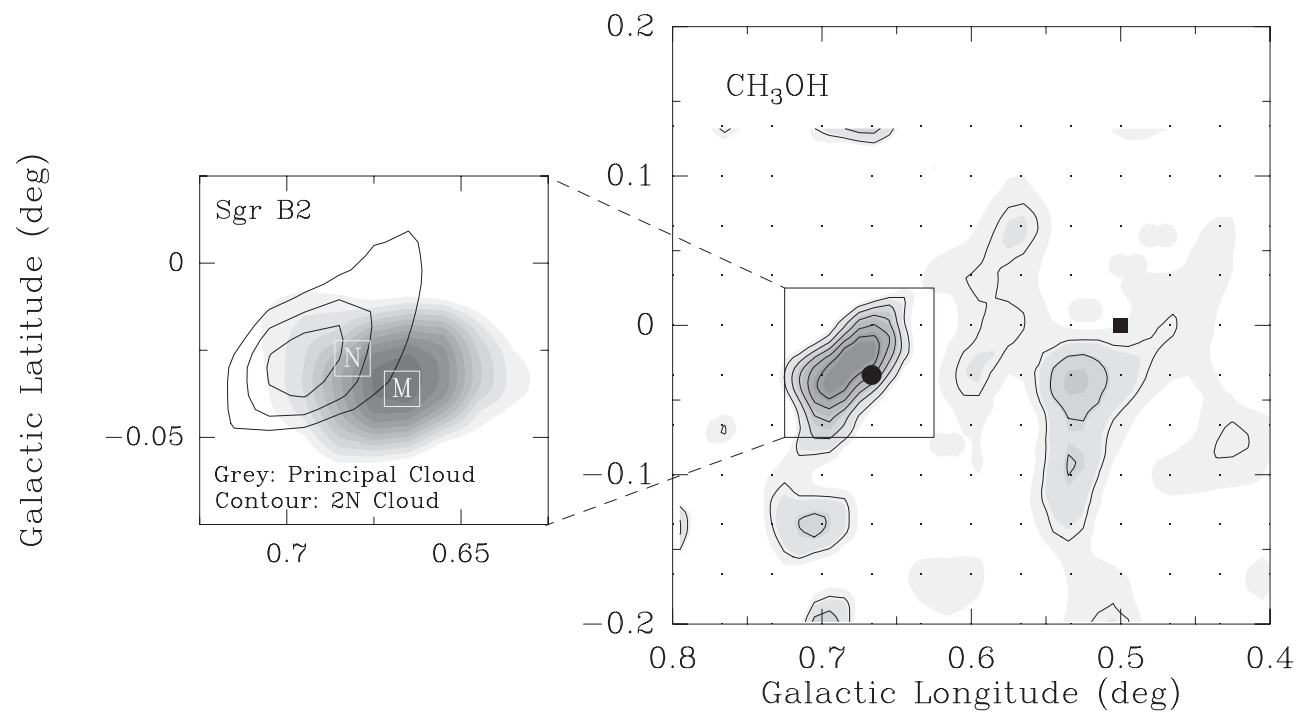

Fig. 2. (Right) Velocity integrated intensity $\left(\int \mathrm{T}_{\mathrm{A}}^{*} \mathrm{dv}\right.$ ) map of the observed $\mathrm{CH}_{3} \mathrm{OH}$ line in the velocity range of $-50 \sim 150 \mathrm{~km} \mathrm{~s}^{-1}$. Contour lines start from $1 \mathrm{~K} \mathrm{~km} \mathrm{~s}^{-1}$ and increase by $1 \mathrm{~K} \mathrm{~km} \mathrm{~s}^{-1}$ for the extended emission except the Sgr B2 (M). The Sgr B1 position is indicated as a filled square, and the small dots are observed points. The data obtained toward the filled circle position, which is roughly the Sgr B2 (M) position, has not been included in making this figure, as explained in Section 3. (Left) Outlines of the Principal Cloud (grey scale, with 10 $\mathrm{K} \mathrm{km} \mathrm{s}^{-1}$ steps between 200 and $300 \mathrm{~K} \mathrm{~km} \mathrm{~s}^{-1}$ for the ${ }^{13} \mathrm{CO} 1-0$ intensity) and the 2'N Cloud (solid line contour, with a $20 \mathrm{~K} \mathrm{~km} \mathrm{~s}^{-1} \mathrm{step}$ from $120 \mathrm{~K} \mathrm{~km} \mathrm{~s}^{-1}$ for the HNCO 4 $0^{-3}{ }_{03}$ intensity) reproduced from Minh \& Irvine (2006). The Sgr B2 (M) and (N) positions are indicated. 


\section{ACKNOWLEDGEMENTS}

This work was supported by Mid-career Researcher Program through National Research Foundation (NRF) grant funded by the Ministry of Education, Science and Technology (MEST) (No. 2009-0083999), and by KRF2008-313-C00376. SJK acknowledges support from the World Class University (WCU) grant (R31-10016) of MEST.

\section{REFERENCES}

Booth RS, Delgado G, Hagstrom M, Johansson LEB, Murphy DC, The Swedish-ESO submillimetre telescope (SEST), A\&A, 216, 315-324 (1989).

Cummins SE, Linke RA, Thaddeus P, A survey of the millimeter-wave spectrum of Sagittarius B2, ApJS, 60, 819-878 (1986). doi: 10.1086/191102

Dartois E, Schutte W, Geballe TR, Demyk K, Ehrenfreund P, et al., Methanol: the second most abundant ice species towards the high-mass protostars RAFGL7009S and W 33A, A\&A, 342, L32-L35 (1999).

Gibb EL, An inventory of interstellar ices toward the embedded protostar W33A, ApJ, 536, 347-356 (2000). doi: $10.1086 / 308940$

Hasegawa T, Sato F, Whiteoak JB, Miyawaki R, 1994, A largescale cloud collision in the Galactic center molecular cloud near Sagittarius B21, ApJ, 429, L77-L80, doi: $10.1086 / 187417$

Irvine WM, Goldsmith PF, Hjalmarson Å, Chemical abundances in molecular clouds, in Interstellar processes, eds. Hollenbach DJ, Thronson, HA, Jr. (Dordrecht, Reidel, 1987), 561.

LaRosa TN, Kassim NE, Lazio TJW, Hyman SD, A wide-field 90 centimeter VLA image of the Galactic center region, ApJ, 119, 207-240 (2000). doi: 10.1086/301168

Leurini S, Schilke P, Menten KM, Flower DR, Pottage JT, et al., Methanol as a diagnostic tool of interstellar clouds. I. Model calculations and application to molecular clouds, ApJ, 422, 573-585 (2004). doi: 10.1051/00046361:20047046

Leurini S, Schilke P, Wyrowski F, Menten KM, Methanol as a diagnostic tool of interstellar clouds. II. Modelling highmass protostellar objects, A\&A, 466, 215-228 (2007). doi: 10.1051/0004-6361:20054245

Lis DC, Serabyn E, Zylka R, Li Y, Quiescent giant molecular cloud cores in the Galactic center, ApJ, 550, 761-777
(2001). doi: 10.1086/319815

Martìn-Pintado J, Gaume RA, Rodrìguez-Fernàndez N, de Vicente P, Wilson TL, Hot expanding shells in the envelope of the Sagittarius B2 molecular cloud, ApJ, 519, 667-686 (1999). doi: 10.1086/307399

Mehringer DM, Menten KM, $44 \mathrm{GHz}$ methanol masers and quasi-thermal emission in Sagittarius B2, ApJ, 474, 346 (1997). doi: 10.1086/303454

Mehringer DM, Palmer P, Goss WM, 6 centimeter formaldehyde absorption toward the Sagittarius B star-forming complex, ApJS, 97, 497-511 (1995). doi: 10.1086/192148

Meier DS, Turner JL, Spatially resolved chemistry in nearby galaxies. I. The center of IC 342, ApJ, 618, 259-280 (2005). doi: $10.1086 / 426499$

Minh YC, Haikala L, Hjalmarson A, Irvine WM, Are clouds collapsing at the 2 north position of Sagittarius B2?, ApJ, 498, 261 (1998). doi: 10.1086/305558

Minh YC, Irvine WM, The HNCO ring in the Sgr B2 region, NewA, 11, 594-599 (2006). doi: 10.1016/j.newast.2006. 03.004

Minh YC, Kim SJ, Pak S, Lee S, Irvine WM, et al, Molecular abundance variations near our Galactic center, NewA, 10, 425-438 (2005). doi: 10.1016/j.newast.2005.03.001

Minh YC, Ohishi M, Roh DG, Ishiguro M, Irvine WM, Interferometric observations for oxygen-containing organic molecules toward Orion-KL, ApJ, 411, 773-777 (1993). doi: 10.1086/172880

Minier V, Booth RS, Conway JE, VLBI observations of 6.7 and 12.2 GHz methanol masers toward high mass star-forming regions. I. Observational results: protostellar disks or outflows?, A\&A, 362, 1093-1108 (2000).

Nummeline A, Bergman P, Hjalmarson Å, Friberg P, Irvine $\mathrm{WM}$, et al., A three-position spectral line survey of Sagittarius B2 between 218 and 263 GHZ. II. Data analysis, ApJS, 128, 213-243 (2000), doi: 10.1086/313376

Oka T, Hasegawa T, Sato F, Tsuboi M, Miyazaki A, A largescale CO survey of the Galactic center, ApJS, 118, 455515 (1998). doi: 10.1086/313138

Rodgers SD, Charnley SB, Chemical evolution in protostellar envelopes: cocoon chemistry, ApJ, 585, 355-371 (2003). doi: 10.1086/345497

Rodrìguez-FernàndezNJ, Martìn-PintadoJ, deVicenteP,Fuente A, The warm molecular gas in the Galactic center, Ap\&SS, 281, 331-332 (2002). doi: 10.1023/A:1019593524808

Sato F, Hasegawa T, Whiteoak JB, Miyawaki R, Cloud collision-induced star formation in Sagittarius B2. I. Largescale kinematics, ApJ, 535, 857-868 (2000). doi: 10.1086/ 308856 